ఠ

\title{
From LCME probation to compliance: the Marshall University Joan C Edwards School of Medicine experience
}

This article was published in the following Dove Press journal:

Advances in Medical Education and Practice

10 October 2014

Number of times this article has been viewed

\section{Bobby Miller \\ Brian Dzwonek \\ Aaron McGuffin \\ Joseph I Shapiro}

Joan C Edwards School of Medicine, Marshall University, Huntington, WV, USA

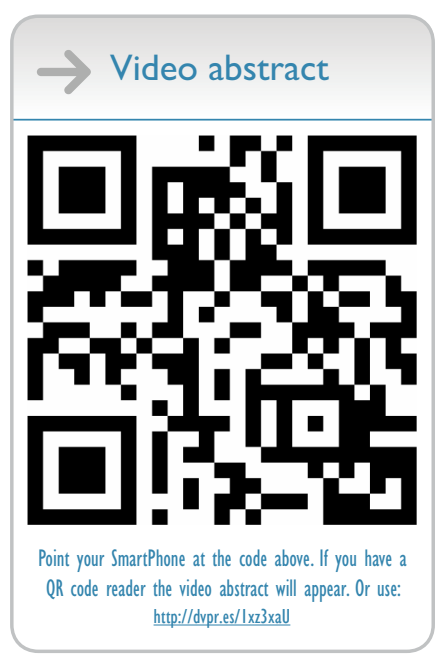

Correspondence: Joseph I Shapiro Joan C Edwards School of Medicine, 1600 Medical Center Drive, Suite 3408, Huntington, WV 2570I-3655, USA

$\mathrm{Tel}+|30469| 1700$

Fax + I 304 69I I726

Email shapiroj@marshall.edu
Abstract: The Joan C Edwards School of Medicine (Marshall University, Huntington, WV, USA) was placed on probation by the Liaison Committee on Medical Education (LCME) in June 2011. In the following 2 years, extensive changes were made to address the numerous citations that resulted in this probation. In October 2013, the LCME lifted probation. In this article, we detail the challenges and solutions identified relevant to our struggle with compliance.

Keywords: accreditation, culture change, leadership, integration, compliance

\section{Introduction}

The Marshall University School of Medicine was formed in the relatively recent past, admitting its first students in 1978. The parent university is one of the oldest in the US, having been founded in 1837 and named for John Marshall, the fourth chief justice of the US.

The formation of the medical school was envisioned as far back as the 1930s, but various considerations made this impractical until the late 1970s when the visionary leadership of Albert Esposito, Robert Hayes, and Robert Coon (among others), and aided by the federal passage of the Teague-Cranston Act, which resulted in the formation of a Liaison Committee on Medical Education (LCME)-approved medical school. The name was changed to the Joan C Edwards School of Medicine (JCESOM) in May 2000. A community-based medical school with strong ties to the Huntington Veterans Administration Medical Center, the JCESOM focuses its efforts on the training of physicians who plan to devote their careers to serve the population of West Virginia and central Appalachia. Over its years of existence, the JCESOM has grown to graduate about 75 students per year.

The JCESOM has enjoyed steady leadership during its history. There have been only four Deans of the JCESOM since the formation of the medical school - including the current Dean. Charles McKown, the fourth Dean of the JCESOM, had served as Dean for nearly 25 years, prior to his moving to the position of Vice President for health affairs in June 2011. As mentioned earlier, the focus of the school has always been to train physicians to serve the region where the school resides, and under McKown's leadership, the school was quite successful toward that end.

Unfortunately, the JCESOM fell short of compliance in a number of areas, which led to the LCME placing the JCESOM on probation in June 2011. In this article, we hope to illuminate the reasons why this occurred as well as detail the strategies that were employed to bring the JCESOM into compliance. 
We would like to stress at the outset, however, that this paper is not meant to criticize any individuals, least of all McKown, who provided visionary leadership for the JCESOM over 25 years and who maintained a firm commitment to its vital mission. Rather, we hope that our story can help other schools avoid the challenges with accreditation that we experienced.

\section{Citations}

Following the submission of a self-study, a full survey visit occurred on March 13-16, 2011. At its meeting on June 7-9, 2011, the LCME voted to place the educational program leading to the medical doctor degree at the JCESOM at Marshall University on probation. Based on the self-study and the site visit, the LCME found that the JCESOM was not in compliance with nine standards, had one standard in compliance with a need for monitoring, and three standards in transition. Six of these 13 standards were among the most commonly cited standards for the LCME from 2004-2009. ${ }^{1}$ The JCESOM citations are listed in Table 1.

\section{Appeal}

In October 2011, the Interim Dean, the Senior Associate Dean of Education, the Associate Dean for Academic Affairs, and a medical student representative from the JCESOM traveled to Chicago, IL, USA, and delivered an appeal in person with supporting documentation to the LCME. We believed, at the time, that we had identified compelling evidence that had not been adequately presented by the self-study or the full survey

Table I LCME findings from full survey visit in March 201 I

\begin{tabular}{lll}
\hline Standard & Description & Finding \\
\hline IS-16 & Diversity & Not in compliance \\
ED-5A & $\begin{array}{l}\text { Lifelong learning } \\
\text { in the curriculum }\end{array}$ & Not in compliance \\
ED-33 & $\begin{array}{l}\text { Horizontal and vertical } \\
\text { integration of curriculum }\end{array}$ & Not in compliance \\
& Career counseling & Not in compliance \\
MS-19 & Debt counseling & Not in compliance \\
MS-23 & Debt and scholarship & Not in compliance \\
MS-24 & support & Not in compliance \\
MS-26 & Personal wellness & Not in compliance \\
FA-5 & Faculty scholarly & \\
ER-9 & productivity & Not in compliance \\
ED-21 & Affiliation agreements & In compliance with \\
& Diversity & need for monitoring \\
IS-1I & Institutional leadership & Standards in transition \\
ER-6 & Resources for clinical & Standards in transition \\
& instruction & Standards in transition \\
MS-37 & Student study space &
\end{tabular}

Abbreviation: LCME, Liaison Committee on Medical Education. visit. Despite this appeal, the LCME decided to uphold its original decision that JCESOM would remain on probation with a focused visit to occur within 12 months.

\section{Response to appeal}

In response to the disappointing outcome of the appeal process, an Interim Dean was selected from the recently retired senior faculty, essentially brought out of retirement, to shepherd the rehabilitation process as well as the search for the new Dean. A steering committee to develop an action plan and address the citations was established on October 19, 2011. Eight subcommittees were formed, based upon the LCME standards felt not to be in compliance (one standard not in compliance concerning affiliation agreements was dealt with administratively). Each subcommittee selected a chairperson and was charged with developing an action plan that would bring the various standards into compliance over the next 12 months. Reasonable and effective action plans were due to the Office of Medical Education (OME) by December 31, 2011. All action plans were reviewed by the steering committee responsible for the oversight of our probationary period in preparation for the LCME secretariat consult visit in January 2012. Final action plans were submitted to the LCME status report shared drive on April 9, 2012. While awaiting approval, the steering committee and subcommittees began implementation of the action plans. On June 22, 2012, the letter was received from the LCME providing approval of the action plan.

\section{Response to probation}

As a result of the probationary status of JCESOM, the leadership of the school approved the new hiring of several key administrative positions including the Dean, Vice Dean of Research, Director of the Marshall Institute of Interdisciplinary Research, and Associate Deans of Diversity and Medical Education. In most cases, these hires occurred after national searches. At the time of the final report to the LCME, searches and offers were made for: a new director for the Edwards Comprehensive Cancer Center; a new permanent chair for the Department of Psychiatry; and a new director for the Division of Endocrinology. These positions have since been filled. Creating and hiring candidates for these positions reinforced a necessary and renewed emphasis on research, diversity, and education - all key areas cited as deficiencies by the LCME.

With these personnel in place, the school's leadership determined the overall strategy of transparency was a key element to establishing a culture of change. 
The most visible and first step in this process was to create a public online JCESOM LCME information page containing all relevant documentation timelines and project plans related to the response to probation. We cannot overemphasize the importance of this step in gaining the input, trust, and support from the alumni, students, faculty, and staff.

Suchman describes organizational culture as being created and maintained by the behavior of individuals and patterns of behavior within an organization. ${ }^{2}$ These behavioral patterns influence how individuals interact and behave within structured and unstructured encounters and directly influence how decisions are made. At JCESOM, the probationary status afforded us the opportunity to address changes to our organizational culture by empowering individuals and committees to influence change. The sense of empowerment created a new sense of teamwork and ownership that was the catalyst for the outcomes described in the final report to the LCME.

During the probationary period, the steering committee composed of the chairperson for each of the eight standards subcommittees, the Dean, and the Senior Associate Dean for education met on a biweekly basis. During these meetings, progress was reviewed and plans further delineated for the subcommittees to work through and complete their action plans. Details, meeting minutes, and the timeline can be viewed at: http://musom.marshall.edu/lcme/index2013.asp.

The outcomes of this work formed the basis of the response to probation and the documentation for the limited site visit. This committee was also responsible for gathering data to support evidence of change, including meeting agendas and minutes and to disseminate these data in formal reports to faculty, staff, students, and the general public. This was carried out through regular faculty meetings, departmental meetings, small group meetings, town hall meetings, websites, social media, email, and one-on-one discussions. A timeline for the specific responses of the medical school to the probationary status is shown in Figure 2.

The surveillance of the subcommittees was carried out primarily by establishing a number of task-specific timelines and deliverables directly related to the action plan. Surveillance and compliance were reinforced through regularly updated project plans that focused on tasks' timelines and deliverables in the form of Gantt charts. These Gantt charts can be viewed on the LCME web page (http://musom. marshall.edu/lcme/index2013.asp) as a tab marked "Project Plan" for each specific standard. A more typical tabular description of the activities specific to the standard can be seen under the tab marked "Progress Update."

During the probationary period, the steering committee reviewed the briefing book contents as a living document, focusing on recent updates on a biweekly basis. Although the subcommittee chairs for the standards were the primary authors of each of these drafts, other faculty, staff, and students contributed to each of them. Moreover, extensive rewriting and editing were performed by the Senior Associate Dean for Medical Education, as well as the Dean. As part of this process, appendices were created with supporting documentation; these were also populated and reviewed throughout the period leading up to the focused site visit. The final briefing book was 70 pages in length, with the appendices

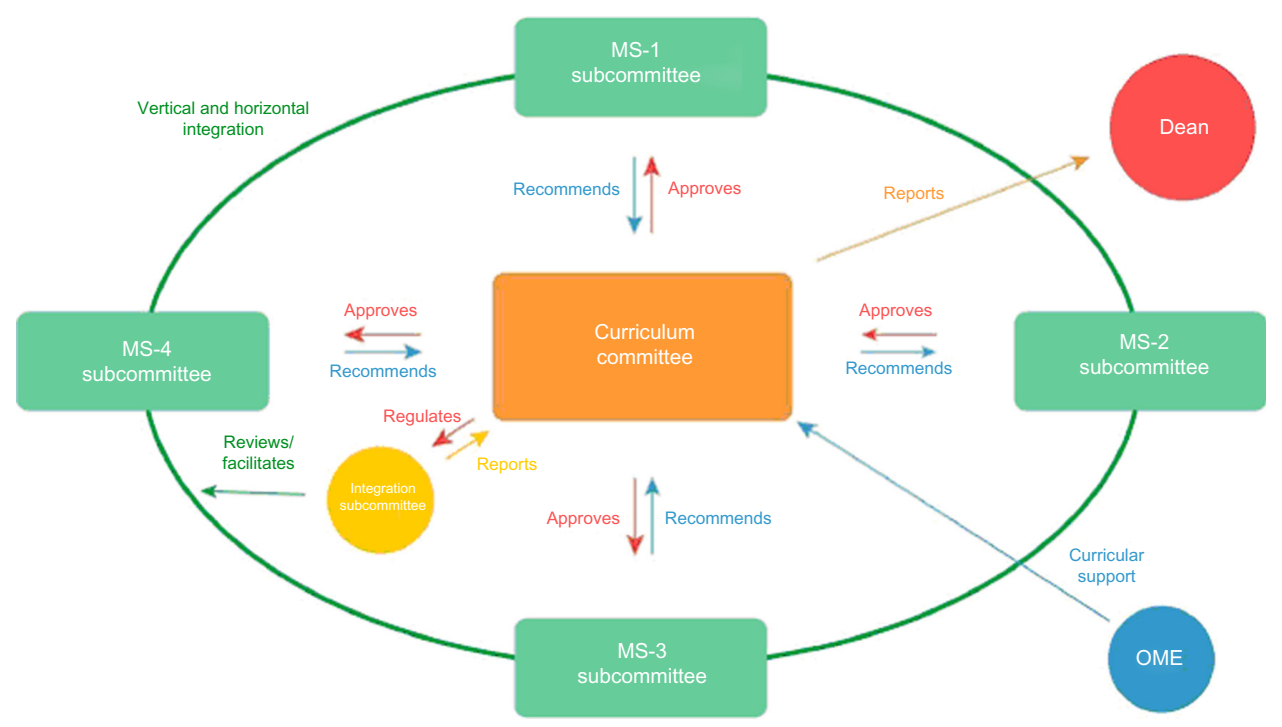

Figure I Schematic showing information flow to and from the curriculum committee to the various subcommittees, the OME, and the Dean. Abbreviation: OME, Office of Medical Education. 
A

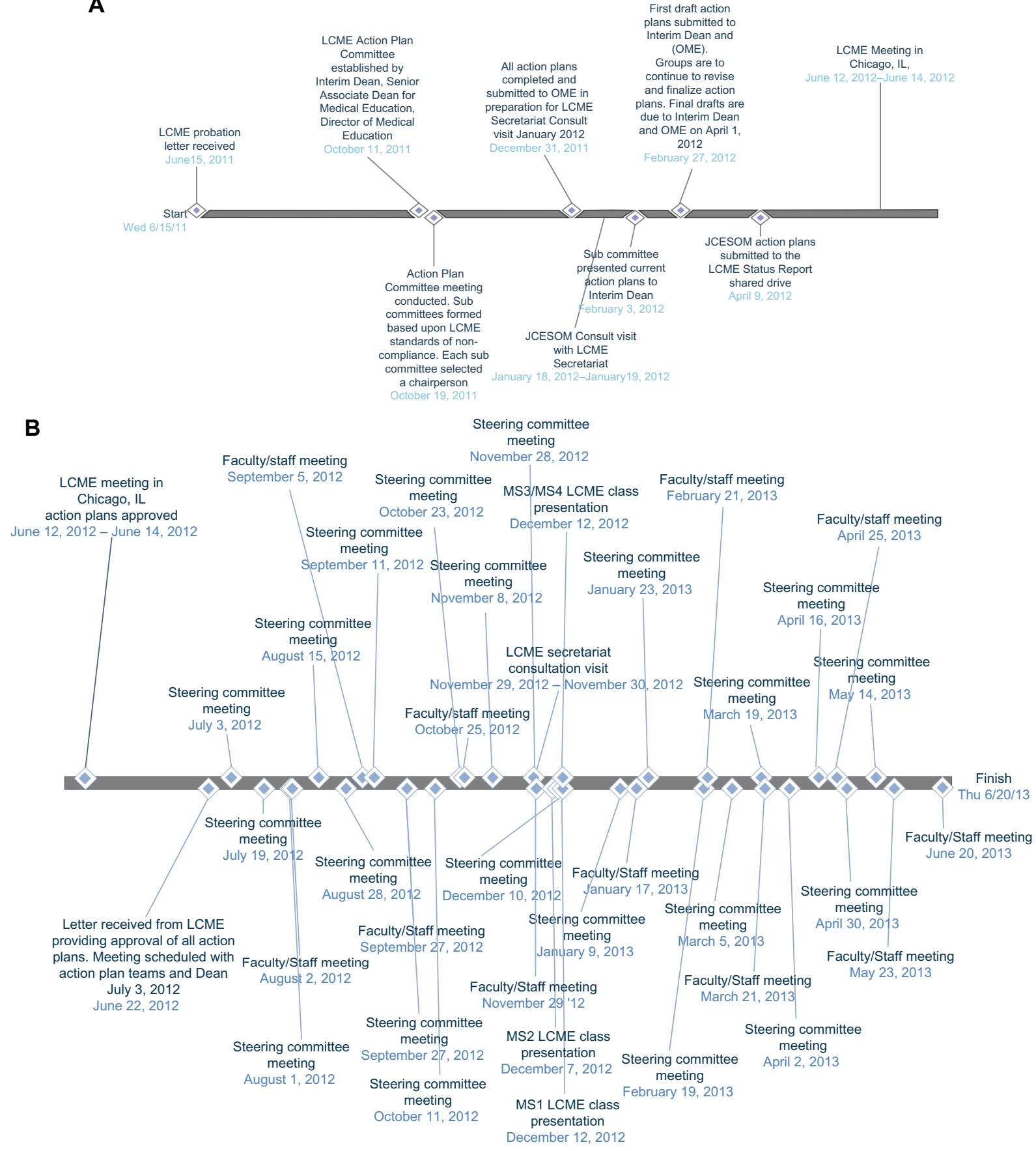

Figure 2 Timeline of events leading to probation and lifting of probation.

Notes: (A) Events following receipt of probation letter in June 201 I prior to LCME meeting in June 20I2. (B) Events after aforementioned LCME meetings leading up to focused site visit by LCME in June 2013 .

Abbreviations: OME, Office of Medical Education; JCESOM, Joan C Edwards School of Medicine; LCME, Liaison Committee on Medical Education.

providing nearly 400 pages of supporting documentation. The briefing book may be viewed in its entirety at http:// musom.marshall.edu/lcme/documents/BriefingBook/ LCME BriefingBook.pdf.

The appendices may be viewed at http://musom.marshall. edu/lcme/documents/BriefingBook/Appendices/. Specific response to the citations is detailed in these documents.
Last, mock interview sessions were held for the various groups selected for review based on the LCME agenda. Feedback on performance was given, strengths and weaknesses were assessed, and the sessions repeated a month later. Participants believed this added to their confidence when the time came for the actual limited (focused) site visit. ${ }^{3}$ Other institutions have described the 
importance of such dress rehearsals in preparing for the actual site visit. ${ }^{4}$

\section{Outcomes}

To appreciate the challenge of changing the culture within a medical education program, we would like to provide you with a brief overview of the outcomes of implementing the action plans as they relate to each standard with an unfavorable finding. These details may be found at: http:// musom.marshall.edu/lcme/index2013.asp.

As ED-33 is both a common and extremely serious standard often cited in cases of probation, ${ }^{1}$ we will discuss our response to this citation. We also provide a flow chart (Figure 1) to represent the current model of curricular management and integration employed at the JCESOM.

As discussed in the LCME standards, the curriculum committee must be in control of the curriculum and, thereby, approve all work and recommendations of the subcommittees. Specifically, the JCESOM curriculum committee reviews and approves and/or designs appropriate changes to the content and pedagogy on a regular basis (should be at least annually). Our curriculum committee also tracks the themes and special topics and recommends appropriate additions to the curriculum (using the curriculum database). The year subcommittees are responsible for the coordination and delivery of the curriculum including horizontal (within the year) and vertical (between the years) integration, pedagogy, and student assessment. The integration subcommittee facilitates this process by reviewing the material to ensure that competencies, diseases, and themes (from the approved list) are integrated appropriately. The integration committee provides regular reports to the Curriculum Committee on integration progress. The OME provides curricular support to the Curriculum Committee and to the various subcommittees.

It is critical to point out that while the curriculum committee continually updates the Dean regarding the curriculum, it is not advisory in this capacity. In fact, the Dean and the OME are advisory to the Curriculum Committee which, as an instrument of the JCESOM faculty, "owns" the curriculum (Figure 1).

\section{Results of site visit and lifting of probation}

After reviewing the report of the LCME survey team that conducted a limited survey of the medical education program on June 23-26, 2013, the LCME voted to continue the program's accreditation for the balance of the current term. The LCME also voted to end the status of "probation."
Table 2 LCME findings following focused survey visit in June 2013

\begin{tabular}{lll}
\hline Standard & Description & Finding \\
\hline IS-16 & Diversity & $\begin{array}{l}\text { In compliance with need } \\
\text { for monitoring }\end{array}$ \\
ED-5A & $\begin{array}{l}\text { Lifelong learning } \\
\text { in the curriculum }\end{array}$ & $\begin{array}{l}\text { In compliance with need } \\
\text { for monitoring }\end{array}$ \\
ED-33 & Horizontal and vertical & In compliance with need \\
& integration of curriculum & for monitoring \\
MS-19 & Career counseling & In compliance with need \\
& & for monitoring \\
MS-23 & Debt counseling & In compliance \\
MS-24 & Debt and scholarship & In compliance with need \\
& support & for monitoring \\
MS-26 & Personal wellness & In compliance \\
FA-5 & Faculty scholarly & In compliance with need \\
ER-9 & productivity & for monitoring \\
ED-2I & Affiliation agreements & In compliance \\
& Diversity & In compliance with need \\
IS-1I & Institutional leadership & for monitoring \\
ER-6 & Resources for clinical & In compliance \\
MS-37 & instruction & for monitoring \\
\hline
\end{tabular}

Abbreviation: LCME, Liaison Committee on Medical Education.

The program's next full survey is scheduled during the 2018-2019 academic year. Based on the report of the limited site visit team, the LCME found that the JCESOM was in compliance with five standards and had eight standards in compliance with a need for monitoring. These standards are listed in Table 2. To address these compliance issues mentioned earlier, the LCME requested that the Dean submit a status report by December 1, 2014, containing specific information detailed in this letter: http://musom.marshall.edu/lcme/documents/20131021 LCMELetter.pdf.

In 2012, the LCME initiated a self-study to reorganize the format of the standards that had been in place since 2012. Prior to accreditation standards reformatting in 2002, 46 out of 108 of LCME actions were severe versus 60 out of 107 afterward. ${ }^{1}$ During the 2-year process, members of the LCME, and the medical education community as a whole, were asked to contribute suggestions for streamlining the standards while reducing redundancy and improving clarity. In many ways, our school benefited from rich dialog between the medical education community and the LCME. The focused discussion that occurred during the monthly LCME Connecting with the Secretariat teleconferences effectively framed the transition between the current standards and the restructured 2014 accreditation standards. The new standards will be in effect for all accreditation activities taking place after July 2015 (www.lcme.org). 
JCESOM joined the initial group of medical schools that chose to participate in the testing of the curricular reporting component of the Accreditation Standards Self-Evaluation Tool for the 2013-2014 academic year. The new LCME format and electronic reporting structure will drive the continuous quality improvement process by identifying gaps well in advance of a LCME site visit. To our collective thinking, a considerable opportunity derives from the data reporting process that will inform the JCESOM leadership as well as the LCME and, therefore, provide a systematic and timely dashboard for ongoing self-study.

\section{Conclusion}

As we reflect on these events, it is very clear (in retrospect) that our institution made several mistakes that led to probationary status. ${ }^{1}$ First and foremost, we became too isolated in our thinking, focusing on the local aspects of our mission to the detriment of the functions necessary for any allopathic medical school. This was (we believe) understandable, given the incredible importance and relevance of our medical school to the WV/Appalachian region, but it still must be avoided in the future. Other problems could actually be considered extensions of this isolation mentality.

That said, the authors of this paper are very proud of the administration, faculty, and staff of Marshall University for making probation into an opportunity to make our school better. Almost every response to the citations was crafted to not only address the citation but also to improve our school. ${ }^{5}$ In fact, we are very optimistic that our school has significantly improved during this process.

This is not to say that process was painless. Very few medical schools are placed on probation, and the embarrassment to our school had non cosmetic consequences. Our class demographics that previously emphasized WV residents changed abruptly (but transiently) in 2012, as we struggled to recruit qualified WV residents to our school. Some faculty recruitments and grant applications during this time were also unsuccessful, and some of this might have been related to our probationary status. It is also fair to point out that the pendulum may have swung too far regarding our responses to probation. The vigorous addressing of citations led to increases in infrastructure costs, which may be difficult to sustain in the current, challenging economic environment. All this said, our school has taken steps to avoid similar accreditation problems in the future, and we sincerely hope that this report of our experiences might help other schools in their own ventures.

\section{Disclosure}

The authors report no conflicts of interest in this work.

\section{References}

1. Hunt D, Migdal M, Eaglen R, Barzansky B, Sabalis R. The unintended consequences of clarity: reviewing the actions of the Liaison Committee on Medical Education before and after the reformatting of accreditation standards. Acad Med. 2012;87(5):560-566.

2. Suchman AL. A new theoretical foundation for relationship-centered care. Complex responsive processes of relating. J Gen Intern Med. 2006;21 Suppl 1:S40-S44.

3. Davis DJ, Ringsted C. Accreditation of undergraduate and graduate medical education: how do the standards contribute to quality? $A d v$ Health Sci Educ Theory Pract. 2006;11(3):305-313.

4. Chandran L, Fleit HB, Shroyer AL. Academic medicine change management: the power of the liaison committee on medical education accreditation process. Acad Med. 2013;88(9):1225-1231.

5. Cottingham AH, Suchman AL, Litzelman DK, et al. Enhancing the informal curriculum of a medical school: a case study in organizational culture change. J Gen Intern Med. 2008;23(6):715-722.
Advances in Medical Education and Practice

\section{Publish your work in this journal}

Advances in Medical Education and Practice is an international, peerreviewed, open access journal that aims to present and publish research on Medical Education covering medical, dental, nursing and allied health care professional education. The journal covers undergraduate education, postgraduate training and continuing medical education

\section{Dovepress}

including emerging trends and innovative models linking education, research, and health care services. The manuscript management system is completely online and includes a very quick and fair peer-review system. Visit http://www.dovepress.com/testimonials.php to read real quotes from published authors. 